Proc. Indian Acad. Sci. (Chem. Sci.), Vol. 105, No. 6, December 1993, pp. 315-331.

(C) Printed in India.

\title{
Strategies for solar fuel generation
}

\author{
HELMUT TRIBUTSCH \\ Hahn-Meitner-Institut, Dept. Solare Energetik, D-14109 Berlin, Germany
}

\begin{abstract}
Solar production and use of fuels (hydrogen, reduced carbon compounds) is an integral part of any large scale solar energy strategy. Technically, the most promising pattern of implementation is the decentralised generation of photovoltaic energy on buildings, interconnection with the public electricity grid and centralized electrolytic generation of fuels. A complementary strategy could be solar electrochemical generation of simple inorganic energy carriers (e.g. $\mathrm{Fe}^{2+}$ from $\mathrm{Fe}^{3+}$ ) which serve as the energy source for bacteria (e.g. Thiobacillus ferrooxidans), which fix carbon dioxide via the Calvin cycle. Direct photoelectrochemical generation of solar fuels (in analogy to photosynthesis) is a complex frontier with many unsolved problems, some of which are discussed. The most promising route appears to be development of photoactive transition metal electrocatalysts, which are able to induce interfacial coordination chemical mechanisms. In addition it may be necessary to develop far-from-equilibrium electrochemical mechanisms to facilitate cooperative electron transfer processes during photocatalysis.

At the long term, solar fuel generation may gradually become economical with increasing consideration of social and environmental costs in the price of fossil energy.
\end{abstract}

Keywords. Solar fuel generation; photovoltaic energy; centralized electrolytic generation; solar electrochemical generation.

\section{Natural and artificial photosynthesis}

The generation of energy-rich chemical compounds, by solar-driven mechanisms in photosynthesis, is the basis for higher forms of life on earth and a model for artificial attempts to produce solar fuels. The photosynthetic process itself is composed of several steps. They include harvesting of light through light-scattering processes and non-imaging optical light concentration, non-radiative energy transfer within assemblies of chlorophyll molecules and the supply of excitation energy to photosynthetic reaction centers. The excited state produces a sensitization-type of electron injection into the electron-transfer chain and electron transfer across the photosynthetic membrane. This electron transport leads to the generation of reduced energy-carrying chemical species (NADPH) and simultaneously also to a proton transfer across the membrane in the opposite direction. The illuminated photosynthetic membrane thus not only produces photoinduced currents and potentials but also behaves like a combined electron- and ion-conductor able to undergo topotactic redox reactions. This means that illumination leads to the insertion and transport of ions. The proton gradient produced between the inside and the outside of the photosynthetic membrane stores energy which is used to drive a proton current across a reversible ATPase which leads to the synthesis of ATP from ADP. The energy-rich compounds ATP and NADPH are then used to fix carbon dioxide for the production of carbon 
compounds as a source of energy and materials. The basis of photoinduced electron transport is the transfer of electrons from water to NADP ${ }^{+}$. This photoinduced watersplitting mechanism leading to the liberation of molecular oxygen and to the formation of the hydrogen species bound to an energy-carrying molecule is the key solar energy conversion process on earth. Life only became possible through evolution of an efficient photocatalytic center for oxygen evolution from water. To evolve one molecule of molecular oxygen four electrons have to be transferred from water. Since radical intermediates have to be avoided for energetic reasons, all four electrons have to be transferred close to the thermodynamic redox potential of $E_{0}=1 \cdot 23 \mathrm{~V}$ (NHE). This is only possible in a highly specialized molecular enzymatic complex, which controls intermediates in an extremely precise way. The water-oxidizing manganese complex in photosynthesis contains two pairs of manganese centers in close proximity. Its exact structure and function is not yet known.

Experimentally, and with synthetic and typically inorganic materials, most elementary steps occurring in photosynthetic membranes can be qualitatively reproduced (Tributsch 1990). They include radiationless energy transfer between dye assemblies, electron injection through sensitization (into oxide semiconductors), conversion of light into electrochemical energy (with semiconductors), photogeneration of reduced species (at semiconductor electrodes), photoinduced ion insertion and transport (at combined

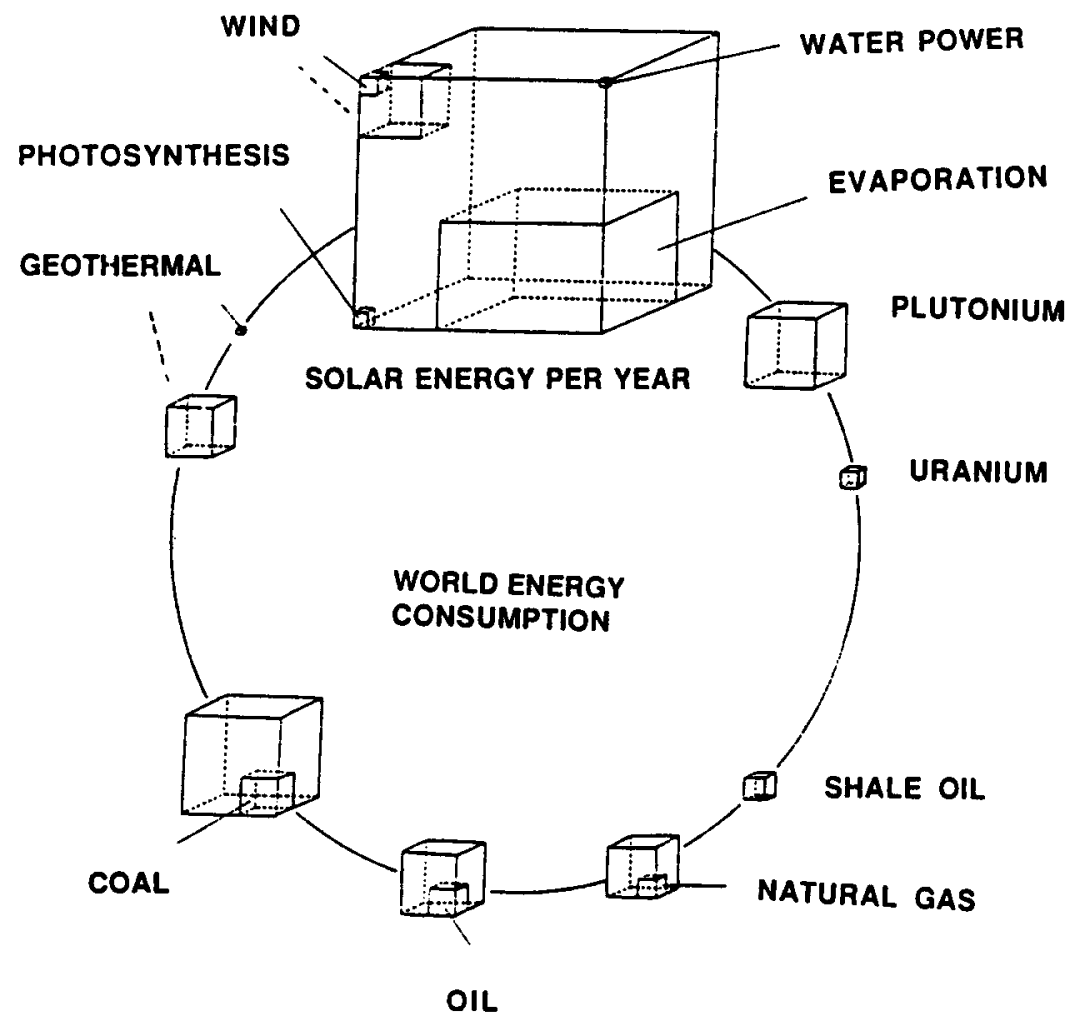

Figure 1. Cubes compare total energy available from different sources with solar energy incident on earth during one year. At the center is shown the world energy consumption. 
semiconducting electron- and ion-conducting materials) as well as photocatalysis of oxygen evolution from water (which up to now is energetically not yet efficient).

The key reason why we cannot yet reproduce photosynthesis artificially for technical applications is our inability to produce stable solid-liquid junctions and to provide complicated photoactive materials at reasonable prices. Since self-organization mechanisms and highly complex bioorganic reactions are not yet technically accessible for artificial photosythesis, simpler technical pathways must be developed. Key elements are inorganic materials, which are thermodynamically and kinetically stable under illumination and polarization. Since cheap and efficient complex-organic catalysts are not available, highly catalytic metals, deposited on semiconductors, must be considered for fuel generation. This means that our inability to deal properly with the complexity and instability of organic molecules and materials forces us to adopt strategies in which the principles of energy conversion are equivalent to the biological ones but the materials are very different. In addition, technical applications aim at much higher energy conversion efficiencies than biological systems, which typically do not specialize in energy conversion but simultaneously also optimize other functions. There is no doubt that large-scale utilization of solar energy is an attractive and reasonable long-term option for mankind. Figure 1 shows that the solar energy incident on earth during one year exceeds the total energy available from all other energy forms, including fossil and nuclear energy via plutonium breeding.

\section{Efficiency considerations}

The most efficient green plants (C4-plants like sugarcane or corn) produce, during their most productive season, biomass with a solar energy efficiency of 2-4\%. Averaged over a farming year, however, not more than $0.5 \%$ solar energy efficiency can be expected. As plants also consume energy for their living processes, the real energy conversion efficiency could be higher by a factor of two. Since a mechanism leading to energy storage always consumes energy, the primary energy conversion efficiency in the most efficient plants may reach $10 \%$ in the most efficient season.

From thermodynamic calculations we know that photon-absorbing materials may produce a solar energy conversion efficiency of up to $31 \%$, depending on their band gap or absorption gap (figure 2). The best evidence that this can reasonably well be approached is given by the highly developed electronic materials silicon and gallium arsenide. Solar energy conversion devices made of silicon with its energy gap of $E g=1 \cdot 1 \mathrm{eV}$ may have a theoretical energy conversion efficiency limit of $27 \%$, while $24 \%$ has been achieved in the laboratory. Gallium arsenide solar cells with an energy gap of approximately $\mathrm{Eg}=1.5 \mathrm{eV}$ have a theoretical energy conversion efficiency of close to $30 \%$ while $27 \%$ has been reached in the laboratory. Several solar energy absorbing materials arranged into a cascade reach theoretical solar energy conversion efficiencies beyond $50 \%$ which suggests that at the long term practical solar energy conversion efficiencies of the order of $40 \%$ may be expected.

Energy is always lost during energy storage in chemicals such as hydrogen. However, for water photoelectrolysis with a tandem solar cell, a theoretical energy conversion efficiency of $27 \%$ and a practical one of $20 \%$ have been estimated (Bolton et al 1985). This means, that well-developed technical solar energy systems for the production of chemical energy could become more efficient by a factor of 40 than agricultural 


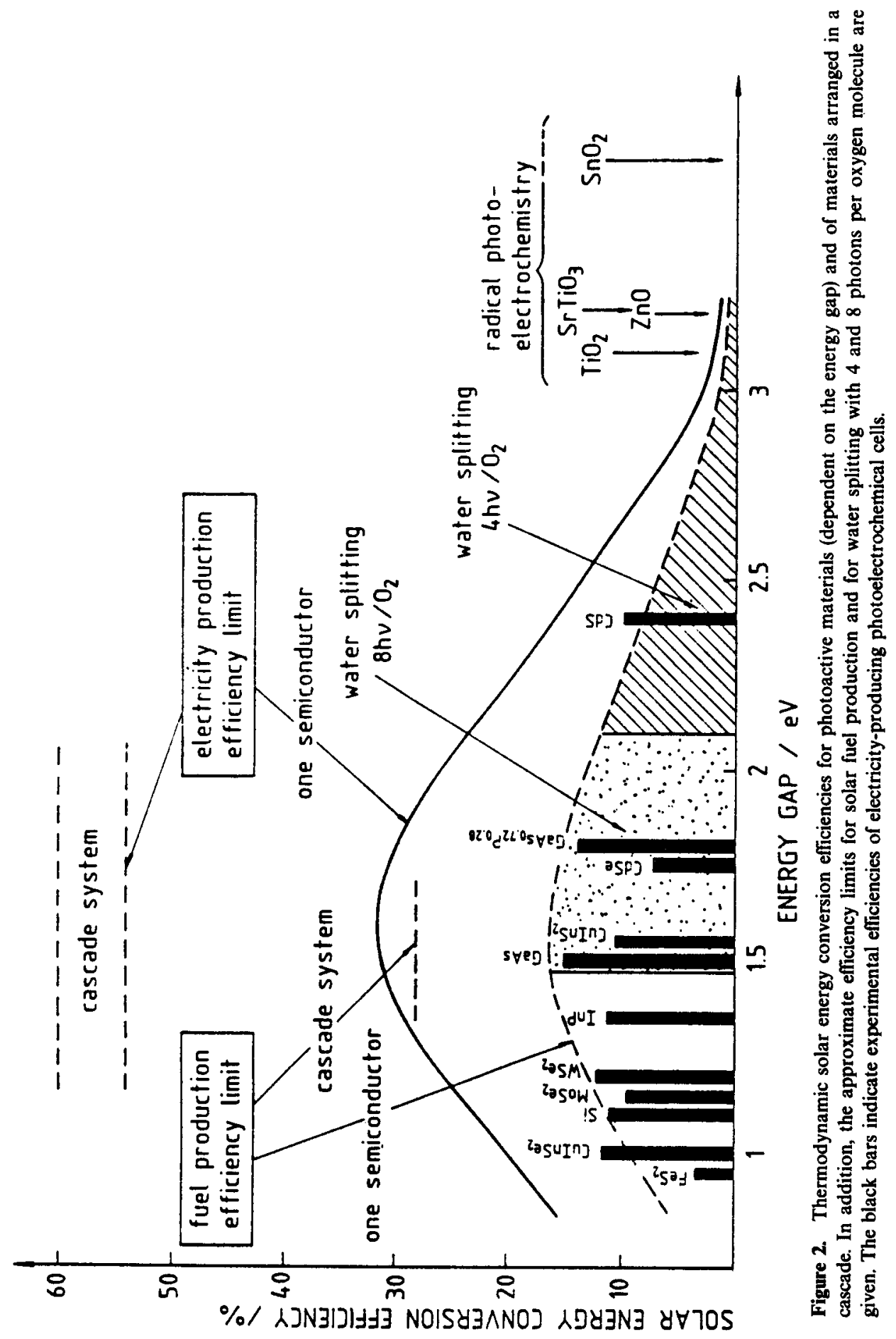


ones $(0.5 \%$ as yearly average). These considerations clearly show the promise of technical systems for solar fuel generation, which should motivate on-going research.

\section{Strategies for solar fuel generation}

\subsection{Photovoltaic-electrochemical generation of hydrogen from water and of carbon compounds from carbon dioxide}

The technically most straightforward way for solar fuel generation is photovoltaic generation of electricity for the production of chemicals by electrochemical syntheses. The classical technical vision of solar pioneers are huge photovoltaic installations in the deserts, which produce electricity for the electrolysis of seawater. A practical obstacle is at present the high cost of photovoltaic electricity which still is ten times as expensive as electricity produced from fossil fuels. However, photovoltaic energy will become cheaper with mass production and improved manufacturing techniques. It may also be expected that social costs of fossil energy, including political and environmental costs, health consequences and greenhouse effects, which are significant, may gradually be added to increase the prize of fossil energy. Recently, the concept of huge centralized photovoltaic power stations has seen a gradual transformation. It became apparent, that - especially in countries with much sunshine - most photovoltaic energy needed could be produced by photovoltaic panels installed on houses, in villages and cities. Small electrical inverters, directly integrated into the panels would convert the direct photocurrent immediately into alternating current which could be coupled into the public electricity grid. In fact, such inverter-equipped panels could directly be plugged in into the grid and during sunshine would only cause the electricity counter to invert the movement and count the negative consumption (equivalent to production). The advantage of such a decentralized, grid-connected photovoltaic system would be that the installation would be simple and flexible and electrical losses would be relatively small. Inconvenient and expensive direct current installations, such as thick cables, could also be avoided. The electrical energy could conveniently be used in centralized installations for hydrogen production for chemical use or for electricity generation in fuel cell power plants (figure 3). Energy self-sufficient solar houses can produce hydrogen directly under pressure to avoid compression for hydrogen storage. A problem, concerning electrode degradation as a consequence of a highly irregular energy supply for water electrolysis could be surmounted by applying a bias potential to the electrodes during intervals without solar energy. Electrode corrosion could in this way be avoided with just a small sacrificial loss of energy.

Electricity can, of course, also be used for direct reduction of carbon dioxide. Recently, much effort has been made to develop metallic electrodes for the generation of alcohol, methane or other chemical energy carriers from carbon dioxide (Azuma et al 1990). Pure copper electrodes turned out to be catalytically the most active material. However, inhibition by intermediates, limited selectivity of the electrodes and toxic side products (carbon monoxide) remain a problem. Additional complications are that relatively high overpotentials have to be applied; that catalysis is limited to comparatively small current densities and that for the applied electrode potentials hydrogen evolution is an efficiency reducing competitive reaction. 


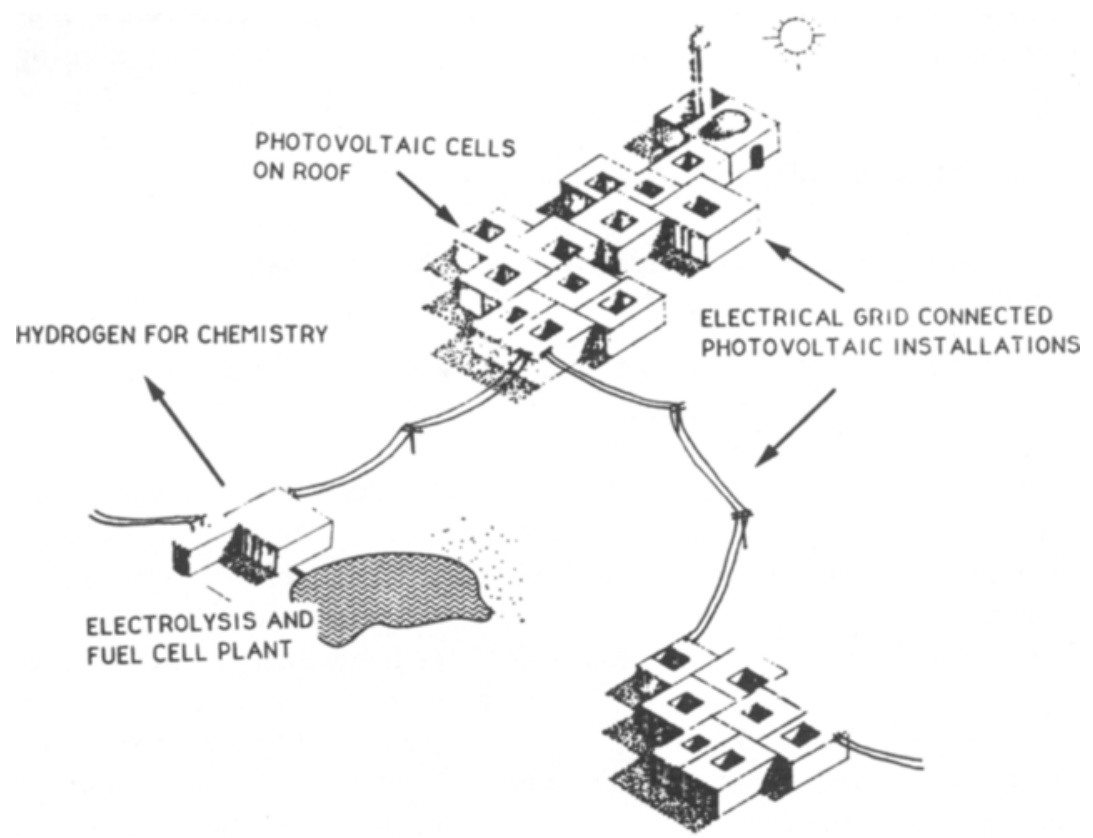

Figure 3. Picture showing decentralized photovoltaic energy production on houses, interconnection with the alternating current grid and centralized hydrogen production and reutilization.

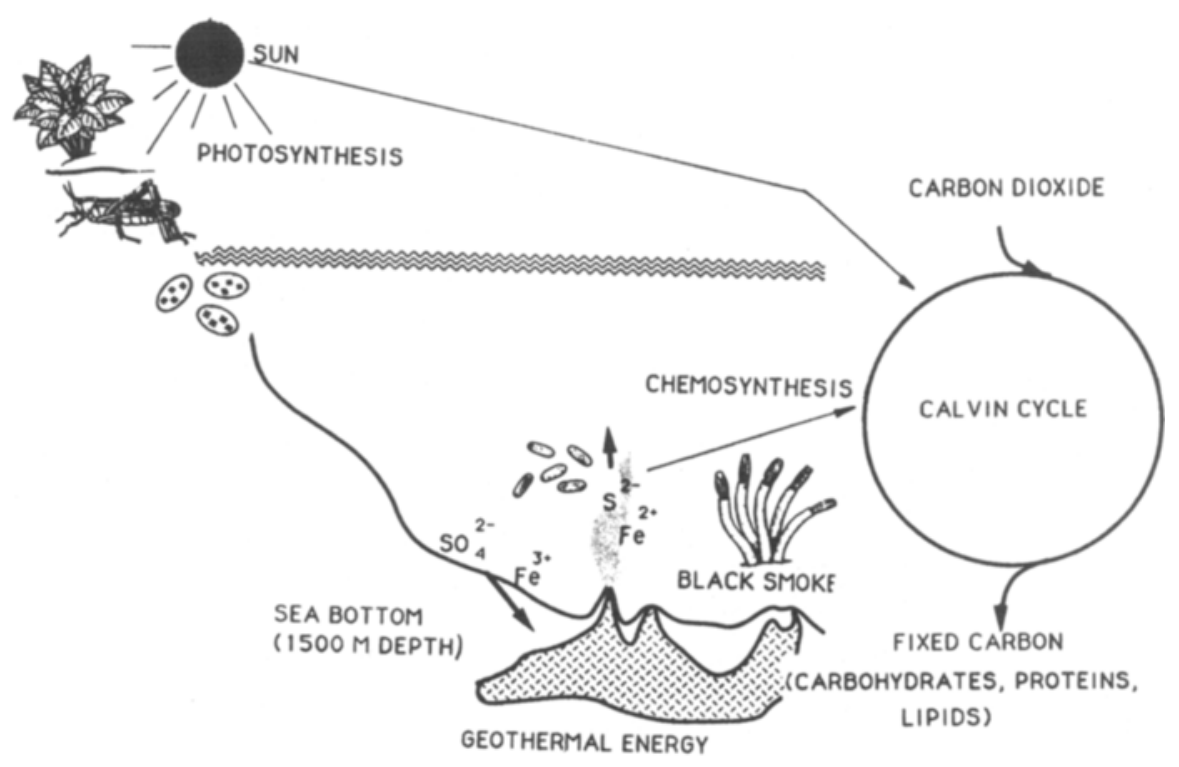

Figure 4. Photosynthesis as well as geothermally powered chemosynthesis can lead to the build-up of carbon compounds via the Calvin cycle. 
In principle, it is possible to isolate biological enzymes which are active in carbon dioxide reduction and to immobilize them on electrodes. But it turns out to be very difficult to keep the immobilized enzymes active for a reasonably long time period.

3.2 Photovoltaic-electrochemical or photoelectrochemical generation of simple energy carriers (e.g. $\mathrm{Fe}(I I)$ from $\mathrm{Fe}(I I I)$, which provide the energy source for carbon dioxide fixating bacteria

Photosynthesis is not the only energy basis sustaining biological ecosystems on earth. Chemosynthesis can also support life and also leads to fixation of carbon dioxide for living activities and biomass formation. Well-known examples are submarine ecosystems existing around so-called black smokers, which are powered by geothermal energy and liberate reduced species (e.g. $\mathrm{Fe}(\mathrm{II})$ ), sulfur and sulfides (figure 4).

Bacteria like Thiobacillus ferrooxidans use the inorganic chemical energy as their only energy source to drive carbon dioxide fixation. In addition they only need a few salts for the supply of chemical elements and an acid environment $(p \mathrm{H}<3)$ to avoid precipitation of $\mathrm{Fe}_{2}\left(\mathrm{SO}_{4}\right)_{3}$. These bacteria are approximately $1 \mu \mathrm{m}$ long, of oval shape and are equipped with a rotating flagellum for propagation. They, in fact, use the same Calvin cycle as plants for carbon dioxide fixation and biomass formation but use inorganic chemical energy instead of photon energy as primary energy source. Depending on whether the bacteria use $\mathrm{Fe}^{2+}$ or a sulfide $\left(\mathrm{FeS}_{2}\right)$ as the energy source, the energy converting chemical reaction can be written as follows.

$$
\begin{aligned}
& 4 \mathrm{FeSO}_{4}+2 \mathrm{H}_{2} \mathrm{SO}_{4}+\mathrm{O}_{2} \stackrel{\text { bact., } \mathrm{CO}_{2}}{\longrightarrow} 2 \mathrm{Fe}_{2}\left(\mathrm{SO}_{4}\right)_{3}+2 \mathrm{H}_{2} \mathrm{O}+\text { biomass, } \\
& 2 \mathrm{FeS}_{2}+7 \mathrm{O}_{2}+2 \mathrm{H}_{2} \mathrm{O} \stackrel{\text { bact., } \mathrm{CO}_{2}}{\longrightarrow} 2 \mathrm{FeSO}_{4}+2 \mathrm{H}_{2} \mathrm{SO}_{4}+\text { biomass. }
\end{aligned}
$$

Biomass formation is based on the fixation of carbon dioxide according to the Calvin cycle:

$$
\mathrm{CO}_{2}+3 \mathrm{ATP}+2 \mathrm{NADH} \rightarrow\left(\mathrm{CH}_{2} \mathrm{O}\right)+3 \mathrm{ADP}+3 \mathrm{P}_{\mathrm{i}}+2 \mathrm{NAD}
$$

Both the energy carriers ATP and NADH are generated by the energy liberated during $\mathrm{Fe}^{2+}$ oxidation $(7.84-5.89 \mathrm{kcal} / \mathrm{mol}$ between $\mathrm{pH} 1.5$ and 3 ) and sulfide oxidation respectively ( $\approx 200 \mathrm{kcal} / \mathrm{mol}$ for a disulfide). $118 \mathrm{kcal} / \mathrm{mol}$ of energy is needed for the reduction of carbon dioxide.

The strategy of using solar energy for the synthesis of simple energy carriers consumed by chemoautotrophic bacteria has been discussed in some detail (Tributsch $1979,1982,1989$ ). In the simplest technical set-up (figure 5), solar energy is used to generate $\mathrm{Fe}^{2+}$ and oxygen in an electrochemical cell either by photovoltaic or photoelectrochemical energy. These two chemical species are sufficient to activate the Calvin cycle via the bacterial metabolism which is able to fix carbon dioxide. $19 \mathrm{Fe}^{2+}$ ions must be oxidized for the fixation of one $\mathrm{CO}_{2}$ molecule. In addition, the bacteria (Thiobacillus ferrooxidans) only need some inorganic minerals and trace elements, which has been confirmed in many cultivation experiments (Kelly et al 1977).

It is thus sufficient to generate $\mathrm{Fe}^{2+}$ and $\mathrm{O}_{2}$ by photovoltaic or photoelectrochemical processes to provide the basis for continuous growth and continuous carbon dioxide fixation by bacterial culture. This process is simple and efficient since the required 


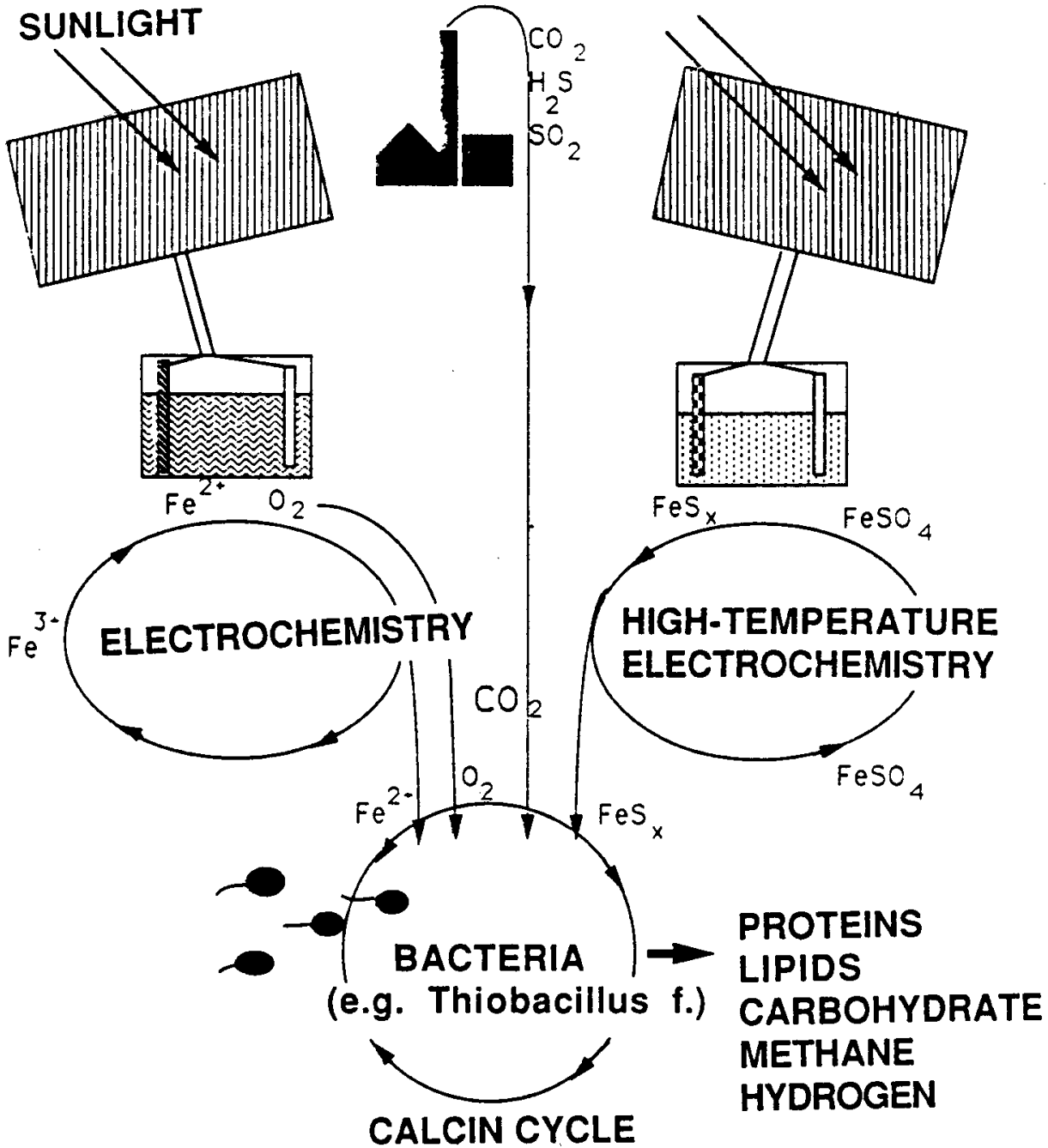

Figure 5. Solar electrochemical and thermoelectrochemical generation of chemical energy carriers $\left(\mathrm{Fe}^{2+}, \mathrm{FeS}_{2}\right)$ for bacterial biomass production.

electrode materials (noble metals and $\mathrm{RuO}_{2}$ ) are available and the bacteria rapidly reproduce in presence of the energy source. The real growth yield (gram dry weight per gram atom $\mathrm{Fe}^{2+}$ ) has been determined for continuous cultures to be 1.33, corresponding to an energy efficiency for $\mathrm{CO}_{2}$ fixation of $\eta_{k}=78.8 \%$ (Kelly et al 1977). Since bacterial life processes themselves consume energy, the real efficiency is reduced to $\eta_{\text {eff }}=47 \%$ (corresponding to a real growth yield of 0.8 ).

Considering an efficiency of $\eta_{e l}=80 \%$ for the photovoltaic generation of $\mathrm{Fe}^{2+}$ in an electrochemical cell, it is found that the total efficiency $\eta=\eta_{k} \eta_{\text {eff }} \eta_{e l}$ for carbon dioxide fixation would be approximately one third of the photovoltaic energy conversion efficiency. This means that for a solar cell with $15 \%$ solar energy conversion efficiency the efficiency of carbon dioxide fixation would be approximately $\eta=5.6 \%$. This is higher than the efficiency of photosynthetic carbon dioxide fixation. If sulfide 
compounds could be synthesized from sulfate by solar-powered electrochemical processes, these sulfides could, of course, also serve as energy carriers for the bacterial energy cycle. The energy density of sulfide/sulfate-based solar-powered biomass production systems would be significantly higher, since the bacteria can gain much more energy from sulfide oxidation.

\subsection{The direct photoelectrocatalytical generation of solar fuels with especially tailored photoactive semiconductor electrodes}

The most fascinating and ambitious strategy for the production of chemicals with solar energy is the direct photoelectrochemical synthesis with catalytic electrodes. It is an interfacial molecular photoelectrochemical approach which follows, with new materials, the principle of the photosynthetic mechanism applied in the thylacoide membranes of plants. In this approach, electrode materials have to be identified, which not only temporarily store excitation energy (semiconductors with an energy gap appropriate for harvesting solar energy), but which also photoreact in a way which facilitates the formation of chemical products which store energy. A key target has been photoelectrochemical water splitting, which can be separated into a cathodic hydrogen-evolution step and an anodic oxygen-evolution step. Hydrogen evolution is a kinetically simple reaction and can quite easily be accomplished using metal catalyst particles (e.g. Pt, Pd, Ni) on photoactive electrodes. Oxygen evolution, however, is a complicated multielectron-transfer reaction and remains the real problem in the water-splitting reaction. Many strategies have been tried to accomplish photoelectrolysis of water including the use of oxides with large energy gaps, of semiconductors which are covered by stable thin-oxide films, catalytic metals or catalytic polymers (for a review see Tributsch 1989b). Much research has been directed into the photoreaction of $\mathrm{TiO}_{2}$ and related large gap oxides (e.g. $\mathrm{SrTiO}_{3}$ ) with water. Even though the photoelectrochemistry of these materials is very interesting, it has not really advanced our knowledge on water splitting with visible light. The oxidation potential (quasi-Fermi level for holes) at $\mathrm{UV}$-illuminated $\mathrm{TiO}_{2}$ interfaces is so positive that $\mathrm{OH}$-radicals can be generated by direct oxidation of $\mathrm{OH}^{-}$. This is the basis of a radical photoelectrochemistry which also allows evolution of oxygen. Such a process is interesting for the degradation of organic waste compounds (Fox 1989), but energetically not favourable for water splitting using visible light.

Our strategy for the photoelectrochemical production of chemicals has been to provide photoelectrodes which allow light-induced interfacial coordination chemical mechanisms to proceed via transition metal states (figure 6). Many transition metal compounds with energy bands derived from transition metal $d$-states have been investigated (table 1). Generally it turns out that an anodic photoreaction with water is possible when photogenerated holes are provided for the interfacial reaction via a semiconductor valence band derived from $d$-states. However, the transition metal must be able to reach a sufficiently high oxidation state for oxygen evolution (e.g. $\mathrm{Pt}, \mathrm{Ru}, \mathrm{Re}$, Ir). If this is not the case as with $\mathrm{Fe}$ in $\mathrm{FeS}_{2}$, the oxygen is transferred to the sulfur for sulfate formation. The oxidation of the chalcogen is favoured when changing from a sulfide to a selenide and telluride (e.g. in $\mathrm{RuX}_{2}$-compounds) due to an increasing overlap of chalcogenide $p$ states with valence band $d$-states.

Water photooxidation to molecular oxygen is efficient when the valence-band edge is made up of pure $d$-states (Jaegermann and Tributsch 1988). There is no doubt that 


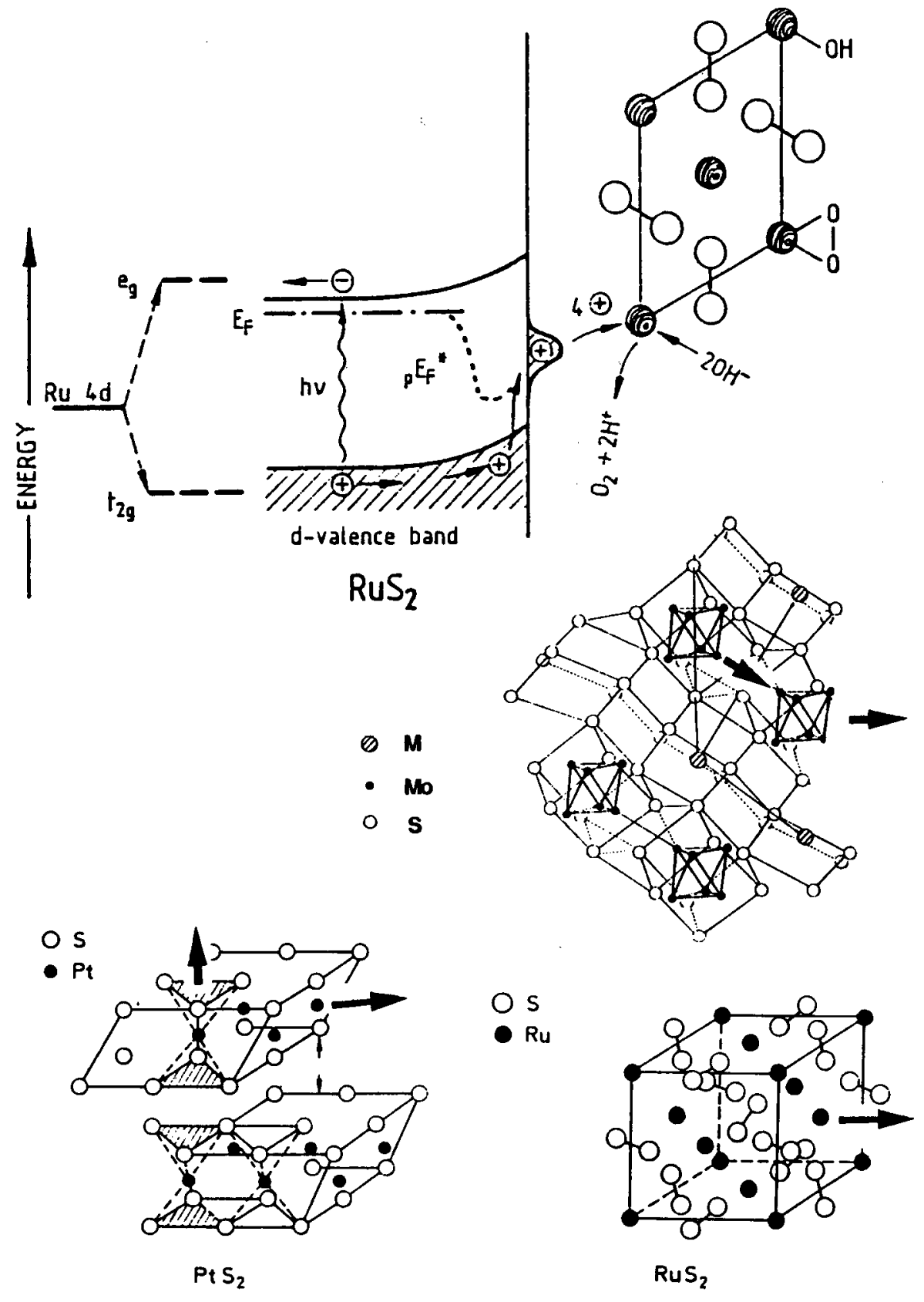

Figure 6. Schematic representation of material research strategy based on $d$-band semiconductors, which permit a photoinduced interfacial coordination chemistry. The path of photoinduced holes (arrows) in layer-type, pyrite-type and cluster-type transition metal compounds is indicated. 
Table 1. Comparison of solid state, chemical and photoelectrochemical properties of different semiconducting $d$-band materials.

\begin{tabular}{|c|c|c|c|c|}
\hline Compounds & $\begin{array}{c}\text { Me-Me } \\
\text { distance } \\
(\AA)\end{array}$ & $\begin{array}{l}\text { Energy gap } \\
\quad(\mathrm{eV})\end{array}$ & $\begin{array}{l}\text { Oxidation state } \\
\text { of metal }\end{array}$ & Photooxidation products \\
\hline $\mathrm{MoS}_{2}$ & $3 \cdot 2$ & $1 \cdot 3$ & Medium & $\mathrm{O}_{2}, \mathrm{MoO}_{4}^{2-}, \mathrm{SO}_{4}^{2-}$ \\
\hline $\mathrm{MoSe}_{2}$ & $3 \cdot 3$ & $1 \cdot 1$ & Medium & $\mathrm{MoO}_{4}^{2-}, \mathrm{SeO}_{4}^{2-}$ \\
\hline $\mathrm{WS}_{2}$ & $3 \cdot 2$ & $1 \cdot 35$ & Medium & $\mathrm{O}_{2}(?), \mathrm{WO}_{3}, \mathrm{SO}_{4}^{2-}$ \\
\hline $\mathrm{WSe}_{2}$ & $3 \cdot 3$ & $1 \cdot 2$ & Medium & $\mathrm{WO}_{3}, \mathrm{SeO}_{4}^{2-}$ \\
\hline $\mathrm{PtS}_{2}$ & 3.6 & 0.95 & High & $\mathrm{O}_{2}$ \\
\hline $\mathbf{R u S}_{2}$ & $3 \cdot 9$ & $1 \cdot 25$ & High & $\mathrm{O}_{2}$ \\
\hline $\mathrm{RuSe}_{2}$ & $4 \cdot 15$ & $<1$ & High & $\mathrm{O}_{2}(90 \%), \mathrm{RuO}_{x}, \mathrm{SO}_{4}^{2-}$ \\
\hline $\mathrm{RuTe}_{2}$ & $4 \cdot 5$ & $<1$ & High & $\mathrm{O}_{2}(10 \%), \mathrm{RuO}_{x}, \mathrm{SO}_{4}^{2-}$ \\
\hline $\mathrm{FeS}_{2}$ & $3 \cdot 8$ & 0.95 & Low & $\mathrm{SO}_{4}^{2-}, \mathrm{Fe}^{3+}$ \\
\hline $\mathrm{IrS}_{2}$ & $4 \cdot 3$ & $\sim 1$ & High & $\mathrm{O}_{2}(? \%)$ \\
\hline $\operatorname{ReS}_{2}$ & $2 \cdot 6-2 \cdot 9$ & 1.4 & High & $\mathrm{Re}_{2} \mathrm{O}_{7}, \mathrm{O}_{2}$ \\
\hline $\mathrm{RuP}_{3}$ & $2 \cdot 8$ & 1.67 & High & $\mathrm{RuO}_{4}, \mathrm{O}_{2}, \mathrm{PO}_{4}^{3-}$ \\
\hline RuPS & $\sim 3.8$ & $1 \cdot 2$ & High & $\mathrm{SO}_{4}^{2-}, \mathrm{PO}_{4}^{3-}$ \\
\hline $\mathrm{Ru}_{2} \mathrm{Mo}_{4} \mathrm{Se}_{8}$ & $\sim 2 \cdot 7$ & $\sim 1 \cdot 3$ & High & $\mathrm{SeO}_{4}^{2-}, \mathrm{MoO}_{4}^{2-}$ \\
\hline
\end{tabular}

a photoinduced interfacial coordination complex is formed with water. Ion-scattering experiments have indeed confirmed that electron donors like $\mathrm{I}^{-}, \mathrm{Br}^{-}$or $\mathrm{OH}^{-}$interact with the interfacial metal centers of the electrode. Attachment of suitable metal ligands to these interfaces (e.g. $\mathrm{CN}, \mathrm{CO}$, pyrazine) improves photoinduced interfacial electron transfer (Schubert and Tributsch 1990; Büker et al 1993) which supports the picture of metal-centered interfacial coordination photoelectrochemistry.

For a long time it remained a puzzle why van der Waals surfaces of layer-type transition metal dichalcogenides with $d$-band structure are photocatalytically active even though the surface-atom layer is made up of chalcogen atoms. Potential-assisted water photooxidation to molecular oxygen has not only been observed for $\mathrm{PtS}_{2}$ (Tributsch and Gorochov 1981), though with a high overpotential typical for platinum, but also for freshly cleaved $\mathrm{MoS}_{2}$ for the first 6-8 hours after starting the experiments. For $\mathrm{MoS}_{2}$ the water oxidation to molecular oxygen, measured by polarographic techniques, gradually changes to molybdenum sulfate formation and corrosion (Tributsch 1977). This latter reaction is facilitated at edge sites of the material, which expose dangling bonds. It may be concluded that water oxidation to molecular oxygen is even possible with a comparable modestly active catalytic metal such as molybdenum, as long as there are no dangling bonds in the environment which could react with intermediate species such as surface-bound oxygen. However, it was unclear how water molecules could have access to the molybdenum $d$-states through a monolayer of sulfur. Recently, high resolution tunnelling electron microscopic pictures performed at $\mathrm{TaSe}_{2}$ have clearly shown that the Mo-dz charge-density contours are visible besides those of the surface sulfur atoms. Atoms at two different levels are sensed by the tunnelling tip. The transition-metal charge density does protrude upward between the sulfur charge density so that it is accessible for reactants from the electrolyte and for chemical interaction (Haneman and Tributsch 1993). 


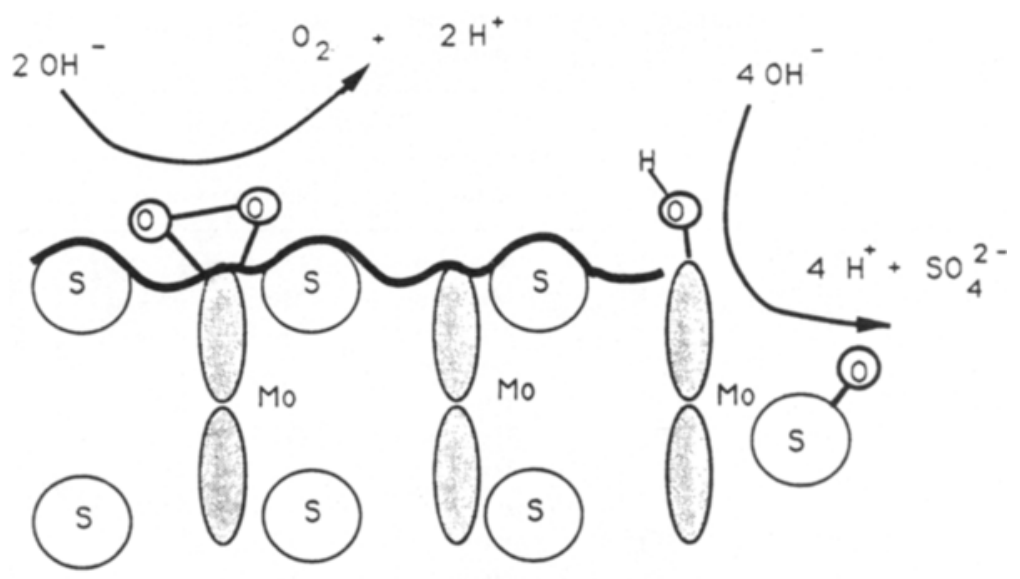

VAN DER WAALS GAP
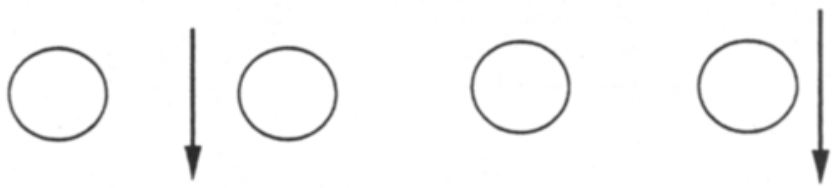

$4 \mathrm{e}-$

$6 e^{-}$

Figure 7. Scheme of charge density profile at van der Waals surface of a layer-type semiconductor $\left(\mathrm{MoS}_{2}, \mathrm{PtS}\right)$ showing accessibility of metal $d z^{2}$ orbitals for interaction with water. An edge site, where corrosion occurs, is also shown.

This explains, why potential-assisted photoinduced oxygen evolution was observed at freshly cleaved $\mathrm{MoS}_{2}$ surfaces. The lack of dangling bonds at the van der Waals surface kept the Mo-peroxo complex sufficiently stable for oxygen evolution.

This confirms that photoelectrochemical interaction of water with transition metals is the key to water oxidation to molecular oxygen. As long as the peroxo-type complex can be stabilized and side reactions are largely suppressed, oxygen may be liberated even with a modestly active transition metal. However, as soon as side reactions with increase of step-site formation (exposed dangling bonds) dominate, sulfate formation and corrosion result. This means that we need not only form a suitable transition metal complex with water photoelectrochemically but have also to provide for a suitable chemical environment. Transition metals which allow the formation of high oxidation states $(\mathrm{Ru}, \mathrm{Pt}, \mathrm{Ir})$ do not seem to readily shift oxygen from their peroxo complex to the sulfur. Therefore photoreactions lead to oxygen evolution when these metal centers are active on $d$-type sulfide semiconductor surfaces. The compound $\mathrm{RuS}_{2}$ turned out to be very efficient for photoinduced potential-assisted oxygen evolution (up to $40 \%$ quantum efficiency observed (Collel et al 1993)) and kinetically very stable (not dissolved by aqua regia). However, when in ruthenium disulfide one sulfur atom is replaced by phosphorus, so that the semiconducting compound RuPS is formed, corrosion is significantly increased (Fleming and Tributsch 1993). The reason may be the ease with which oxygen can be transferred to phosphorus to form soluble phosphate. The molecular basis of interfacial photoreactivity of $\mathrm{RuS}_{2}$ for 
water oxidation to molecular oxygen is presently investigated with new technique and with systematic material modifications. It turns out that the electrical field is the Helmholtz-layer contributes to the efficiency of oxygen evolution (Alonso Vant et al 1993). Electronically degenerate samples which produce a significant potentia drop in the double layer show improved oxygen evolution, which indicates that : potential-dependent activation barrier may be controlling the reaction. Since proto exchange between the surface and the electrolyte participates in the oxygen-evolutios process, this ion-transfer mechanism (e.g. desorption of protons as steps leading $t$ the formation of an interfacial peroxo complex) may be rate determining (Alons Vante et al 1993). The requirement of an interfacial electrical field for photoinduce water oxidation would be an important restriction for semiconducting oxyge: evolution electrodes.

To improve the catalytic activity of materials for photoinduced multielectron transfer, semiconducting $d$-band materials were developed which provide clusters $c$ transition metals as reservoirs and interfacial coordination centers for reactant (Alonso Vante and Tributsch 1986). Most of these are of the type $\mathbf{M}_{X} \mathbf{M o}_{6-X} \mathrm{X}_{4}$ $\mathrm{MMo}_{6} \mathrm{X}_{8}$, or $\operatorname{Re}_{6} \mathrm{X}_{8} \mathrm{Y}_{2}(\mathrm{M}=$ metal; $\mathrm{X}=\mathrm{S}, \mathrm{Se} ; \mathrm{Y}=\mathrm{Cl}, \mathrm{Br})$ and permit insertion $\mathrm{C}$ additional metal atoms into channels between transition metal clusters. At suc interfaces with clustered transition metal centers, interfacial complexes, which ca accommodate several electrons needed for multielectron-transfer catalysis, ar expected. It turns out that oxygen-reduction and hydrogen-evolution in acid solutio at semiconducting electrodes of the type $\mathrm{Ru}_{2} \mathrm{Mo}_{4} \mathrm{Se}_{8}$ are as efficiently catalyzed a at platinum electrodes (Alonso Vante and Tributsch 1986; Alonso Vante et al 1989 A systematic variation of metals inserted into cluster materials showed that cooperativ interaction between metal atoms is important for multielectron transfer. Howeve the proximity of another atom is not important for hydrogen evolution, since th individually most active catalytic element, present in the semiconductor interface, involved in hydrogen evolution catalysis.

Even though cluster compounds with reasonably good photoeffects could $t$ developed and have demonstrated photoinduced oxygen-reduction behaviour (Fisch et al 1993) with high photocatalytic activity, they have not yet shown sufficier stability for anodic photoprocesses. It turned out that anodic metal oxide formatio by less noble metals (e.g. Mo) leading to soluble oxidation products is a limiting sic reaction. Cluster compounds, on the other hand, now already appear to be catalyticall very favourable for cathodic multielectron reactions.

Interfacial coordination chemical mechanisms may be a precondition for phots electrolysis of water but cannot guarantee a low overpotential. The overpotenti: needed will largely be determined by the energetic position and the stepwise dynam transformation of electronic states of the interfacial coordination complexes forme However, transition metal clusters serve as reservoirs for electrons and the energet variation of states in complexes formed during multielectron transfer with these more restricted, which is an energetic advantage.

3.4 Irreversible, nonlinear thermodynamics - a key to efficient kinetics and stabili of fuel producing interfaces?

Several arguments indicate that autocatalysis and dynamic structural changes durir electron transfer are additionally needed for energy efficiency during multi-electrc 


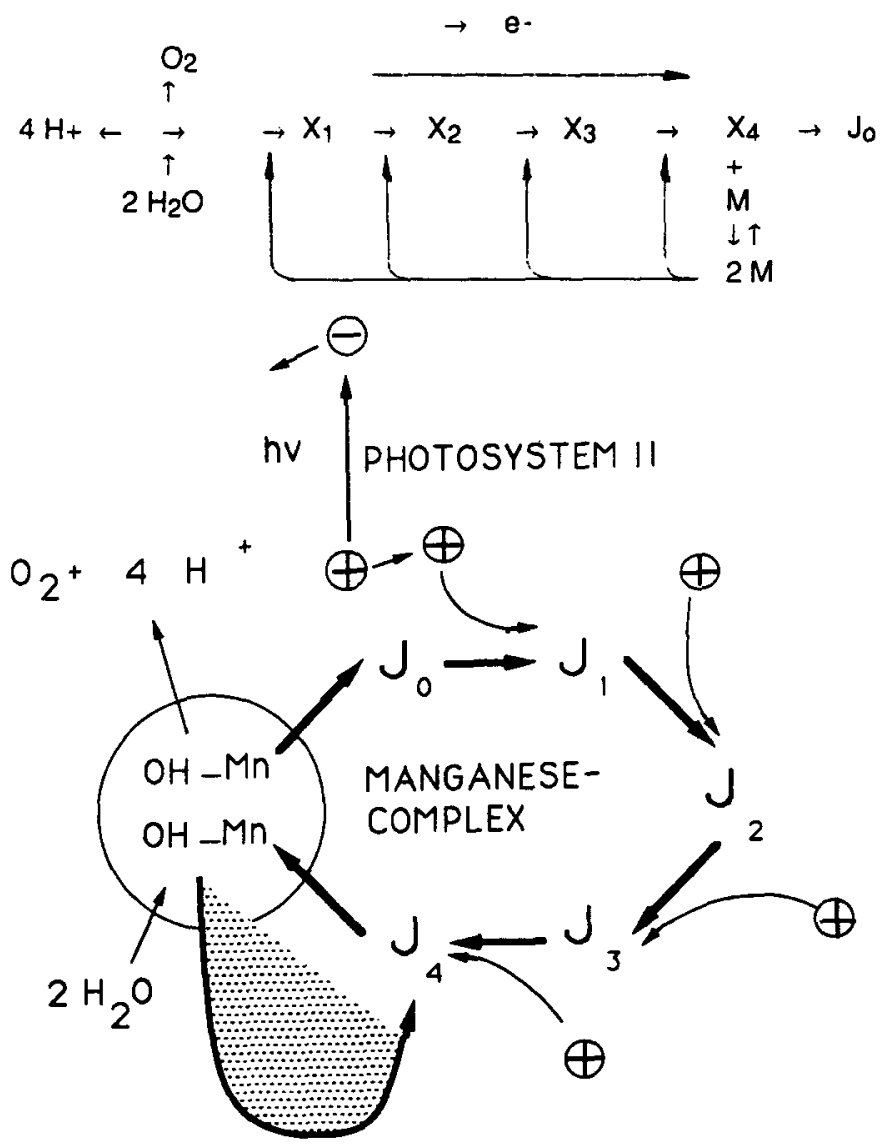

AUTOCATALYSIS

Figure 8. Reaction pathway with nonlinear autocatalytic feedback (above) and proposed function of the manganese-containing oxygen-evolution center of photosynthesis (below).

transfer reactions. In order to avoid successive transfers of electrons with all the difficulty of controlling the energetic properties of intermediates, it may be attempted to transfer electrons collectively in a cooperative way. This concept is new and goes beyond classical electron transfer theory, which only knows successive electron transfer steps. A key element of cooperation within the framework of irreversible thermodynamics is autocatalysis. When the first electron is transferred it must form an intermediate state which exerts a feedback upon the second, third or following electrons. Model calculations indeed show that a "slaving" of degrees of freedom can occur which theoretically proves cooperative electron transfer (Pohlmann and Tributsch 1992; Tributsch and Pohlmann 1992). It can intuitively be understood in analogy to photon emission, which by feedback mechanisms (stimulated emission) turns into coherent laser light. Calculations show that onset of cooperation is accompanied by a breakdown of the activation barrier. In practice, this irreversible thermodynamic approach to multielectron transfer and chemical fuel generation means that very specific electronic-dynamic conformation changes must occur during the process. It may perhaps proceed in the above-mentioned cluster compounds during multielectron 


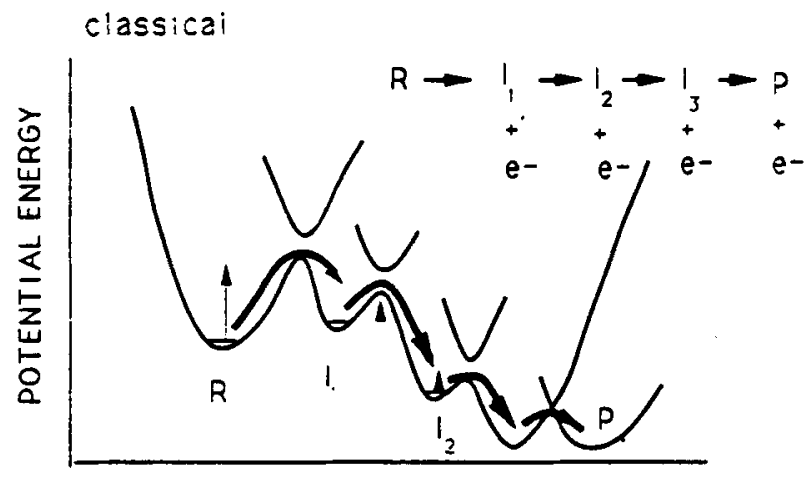

NUCLEAR CONFIGURATION

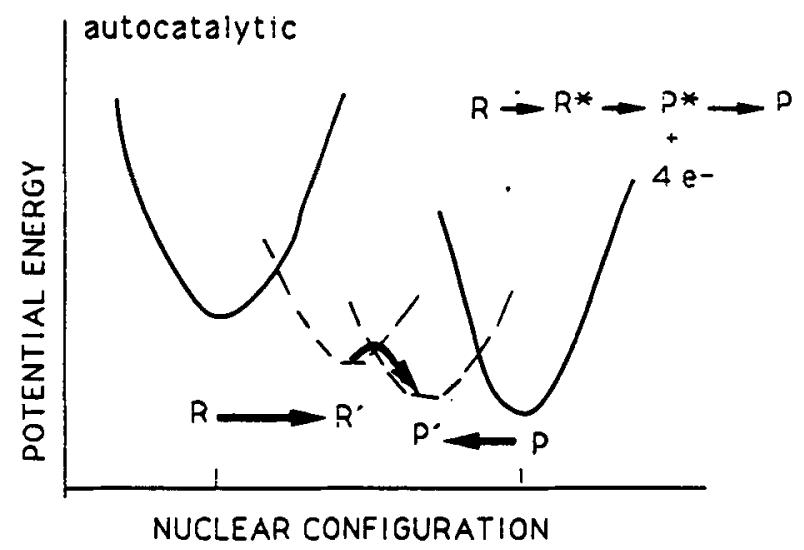

Figure 9. Potential energy-nuclear configuration diagrams of multielectron transfer reaction for classical (above) and proposed cooperative electron transfer (below).

transfer since the volume of the clusters is increased when electrons are extracted. Model calculations show that nonlinear feedback is needed for cooperative electron transfer.

Water photoelectrolysis - as it occurs in photosynthesis - is an energetically extremely efficient process since it occurs very close to the reversible thermodynamic oxidation potential of water $\left(E_{0}=1.23 \mathrm{~V}\right)$ and may thus be considered to be a phenomenon of nonlinear irreversible thermodynamics. The accumulation of four photogenerated holes in the manganese complex may proceed in the conventionally described way, however, during transition of the state $J_{4}$ to $J_{0}$ (which is accompanied by oxygen evolution), the first electron extracted from water must induce an autocatalytic feedback inducing an increase of the transfer rate of the second and the following electrons for inducing cooperative electron transfer. A key problem in this new approach to multielectron transfer catalysis is to understand and properly handle autocatalytic reactions.

Figure 9 compares classical stepwise multielectron transfer with cooperative electron transfer triggered by nonlinear autocatalysis. In this way new nuclear configurations for both the reactant and the product are formed which dramatically reduce activation barriers. It can be imagined that an autocatalytic configuration pathway can be exploited to reach a much more favourable electron-transfer situation. 
Cooperation in catalysis of fuel production may not be the only irreversible process of a new kind to be explored for solar fuel production. It is well known that biological membranes are unstable when extracted from biological systems and it is well known that artificial photoelectrochemical cells have problems with instability. The key strategy which nature employs to stabilize fragile structures is the export of entropy (increase of order) through cooperative mechanisms, based on autocatalytic processes. The entropy of a critical system is maintained by channelling entropy production of the irreversible system through uncritical pathways and by repairing damage. We call the phenomenon self-organization. We may have to learn about it to handle the stability problems of photoelectrodes.

\section{Discussion}

We have seen that initiatives towards solar-fuel production meet several frontiers. One is of political nature. As long as fossil fuels are sold at such low prices without taking into account the real social costs and long-term environmental degradation including the greenhouse effect, fuel from solar energy will not be able to compete. We should nevertheless make efforts to develop solar-fuel technologies because sooner or later mankind will realize that it is not possible to cheat nature and time. Fossil fuels are in fact "borrowed biomass", for which we should pay interest because accumulating carbon dioxide will have to be recycled by photosynthetic processes. This interest (or carbon dioxide tax), should be used to counteract the greenhouse effect by reforestation, energy-efficient architecture, introduction of solar energy technology and energy saving.

The second frontier is a strategic one. Transition to solar energy utilization should be a gradual one and should, to a large extent, guarantee the advantages of modern technology. It should also not threaten the survival of cities with high densities of population. It is my feeling that the most convenient approach to large scale solar fuel production would be the strategy sketched in figure 3: Photovoltaic systems installed in large numbers on buildings, over otherwise-used land and in small photovoltaic plants, which are interconnected with the public electricity grid, will supply most of the electrical energy. Surplus electrical energy will be extracted from the grid in special electrolysis plants for hydrogen production. This hydrogen can be stored to be reconverted to electrical energy via fuel cells. It can also be used for the chemical industry or its energy stored in the form of methanol or ammonia after combining it with carbon dioxide and nitrogen respectively for further use.

An alternative and complementary strategy for chemical-fuel generation from solar energy could be based on the photovoltaic electrochemical generation of a simple inorganic energy carrier such as $\mathrm{Fe}^{2+}$ (from $\mathrm{Fe}^{3+}$ ). In the presence of oxygen it is the only energy carrier needed for carbon dioxide fixating bacteria, which provide high quality biomass containing proteins, carbohydrates and lipids. The solar energy efficiency of this biomass route based on chemosynthetic bacteria exceeds natural photosynthetic energy conversion efficiency. This strategy, which can also be based on a sulfide-sulfate energy cycle, could in fact become the background of an entirely artificial type of agriculture, practicable on infertile desert land.

The third frontier is the microscopic, molecular photoelectrochemical one, which aims at direct photoinduced water splitting or carbon dioxide fixation. This route 
closely follows the strategy applied in green plants, but must, for the near future, rely on significantly less complicated materials and processes. Much research is still needed to produce energy-storing chemicals through such direct interfacial photocatalytical mechanisms. We have shown that a material research strategy towards photoelectrodes able to induce interfacial coordination chemical reactions with water or other reactants for multielectron transfer mechanisms is very promising. Water can indeed be photooxidized to oxygen with high quantum efficiency. However, the overpotential cannot easily be controlled and limited to low values.

Here we have introduced a new strategy to improve the kinetics of fuel generation. The feasibility of cooperative electron transfer has been theoretically demonstrated. The catalyst must be able to exert feedback reactions on subsequent electron transfer steps during electron transfer. This means that it must have the property to induce autocatalytic processes. Such mechanisms belong to nonlinear irreversible thermodynamics. This is a field we consider of great challenge for photoelectrochemistry since it provides mechanisms which can extract entropy or increase order locally at the expense of general entropy production. Entropy export would be helpful both for stabilizing energy-converting electrode interfaces and for the photosynthesis of complicated molecules. Nature has skilfully exploited the potential of far-fromequilibrium mechanisms and it may be that for improved solar generation of fuels we also will have to learn to handle them.

\section{References}

Alonso Vante N, Collel H and Tributsch H 1993 J. Phys. Chem. 97738

Alonso Vante N, Schubert B and Tributsch H 1989 Mater. Chem. Phys. 22281

Alonso Vante $N$ and Tributsch H 1986 Nature (London) 323431

Azuma M, Hashimoto K, Hiramoto M, Watanabe M and Sakata T 1990 J. Electrochem. Sac. 1371772

Bolton R J, Strickler S J and Conolly J S 1985 Nature (London) 316495

Büker K, Vante N A and Tributsch H 1993 (to be published)

Collel H, Alonso Vante $\mathrm{N}$ and Tributsch $\mathrm{H} 1993$ (to be published)

Fischer C, Alonso Vante N and Tributsch H 1993 (to be published)

Fleming I G and Tributsch H $1993 \mathrm{~J}$. Electrochem. Soc. (to be published)

Fox M A 1989 In Photocatalysis (eds) N Serpone and E Pelizetti (New York: J Wiley \& Sons) p. 431 (see also additional contributions in this volume)

Haneman D and Tributsch H 1993 Chem. Phys. Lett. (in press)

Jaegermann W and Tributsch H 1988 Prog. Surf. Sci. 291

Kelly D P, Eccleston M and Jones C A 1977 In Conference on Bacterial Leaching (eds) H G Schlegel and J Barnea (Göttingen: Verlag Chemie)

Pohlmann L and Tributsch H 1992a J. Theor. Biol. 15663

Pohlmann L and Tributsch H 1992b J. Theor. Biol. 199443

Schubert B and Tributsch H 1990 Inorg. Chem. 295042

Tributsch H 1977 Z. Naturforsch. 32361

Tributsch H 1979 Nature (London) 281555

Tributsch H 1982 Aeronaut. Astronaut. 206

Tributsch H 1989a in Photoconversion processes for energy and chemicals (eds) D O Hall, G Grassi (New York, London: Elsevier) p. 283

Tributsch H 1989b In Photocatalysis (eds) N Serpone and E Pelizetti(New York: J Wiley \& Sons) p. 336

Tributsch H 1990 In Proceedings of the 1st World Renewable Energy Conference, Energy and the Environment in the 1990s (ed.) A M Sayigh, vol. 3, p 1447

Tributsch H and Gorochov O 1981 Electrochim. Acta 27215

Tributsch H and Pohlmann L 1992 Chem. Phys. Lett. 188338 